\title{
Spin-triplet pairing instability of the spinon Fermi surface in a $U(1)$ spin liquid
}

\author{
Victor Galitski ${ }^{1}$ and Yong Baek Kim² \\ ${ }^{I}$ Department of Physics and Joint Quantum Institute, University of Maryland, College Park, MD 20742-4111 \\ ${ }^{2}$ Department of Physics, University of Toronto, Toronto, Ontario M5S 1A7, Canada
}

\begin{abstract}
Recent experiments on the organic compound $\kappa-(E T)_{2} C u_{2}(C N)_{3}$ have provided a promising example of a two dimensional spin liquid state. This phase is described by a two-dimensional spinon Fermi sea coupled to a $U(1)$ gauge field. We study Kohn-Luttinger-like pairing instabilities of the spinon Fermi surface due to singular interaction processes with twice-the-Fermi-momentum transfer. We find that under certain circumstances the pairing instability occurs in odd-orbital-angular-momentum/spin-triplet channels. Implications to experiments are discussed.

PACS numbers: 71.27.+a, 71.10.Hf, 71.30.+h
\end{abstract}

Understanding possible phases of matter in strongly interacting electron systems is one of the central issues in condensed matter physics. A prime example of such systems is the Mott insulator. Among a plethora of possible insulating phases, perhaps the most interesting states are spin liquids with no long range order. The possibility of such states of matter was first discussed by Pomeranchuk back in 1941 [1], who conjectured that in insulators, the elementary excitations may be charge-neutral fermions (spinons). More precise and modern form of such proposals had to wait until Anderson proposed the spin liquid state as the key paradigm in high- $T_{c}$ cuprates physics [2].

While the relevance of spin liquid phases to the high$T_{c}$ problem is still under debate, the search for spin liquids has continued in other classes of strongly interacting electron systems. One of the most notable examples is a series of recent experiments on the organic compound $K$ $(E T)_{2} \mathrm{Cu}_{2}(C N)_{3}[3,44]$. Here the electrons are half-filled on the triangular lattice and the relative interaction strength is controlled by applying a hydrostatic pressure. The insulating phase of this system exhibits finite uniform susceptibility and finite specific heat coefficient at low temperatures, i.e. metallike behaviors. It was proposed that the $U(1)$ spin liquid phase with a spinon Fermi surface is a viable explanation of the experimental results [5, 6]. Variational calculations on the Heisenberg-ring-exchange model are consistent with this expectation [5].

The spinon Fermi sea, however, is strongly coupled to a $U(1)$ gauge field. On the lattice, the $U(1)$ gauge field is compact and in principle one should worry about possible confinement effect in two dimensions due to monopole events [7]. It has been argued that the monopole events are suppressed due to the coupling to the gapless spinon degrees of freedom [8, 9]. Nevertheless, the gauge field still gives rise to singular renormalization of various physical quantities. For example, the specific heat coefficient should diverge as $T^{-1 / 3}$ $[10,11,12,13,14]$. This behavior has not been seen and instead there exist abrupt changes in the susceptibility as well as in the specific heat around a few Kelvin [3, 4]. This may suggest a thermodynamic transition or possibly a crossover. It is therefore interesting to consider possible instabilities of the corresponding spinon Fermi sea state.
The instability of the spinon Fermi surface may also be a very useful avenue to study the emergence of other possible phases. Notice that in Fermi liquid theory, all broken symmetry states of ordinary metals can be understood as an instability of the Fermi surface. In the same spirit, the spinon Fermi surface state may be regarded as a mother state of various possible phases of Mott insulators.

In this paper, we study the Kohn-Luttinger-like pairing instabilities [15] of the spinon Fermi surface. Due to the coupling to the gapless gauge field, the spinon interaction vertex for the momentum transfer of twice the Fermi momentum diverges as a power law [12]. This singularity renormalizes the effective interaction in the Cooper channel, which becomes attractive for odd orbital angular momenta. It is found that under certain conditions the system is unstable to the spinon paired state in odd orbital angular momentum (spin-triplet) channels. When the pairing is present, the ground state would be a $Z_{2}$ spin liquid.

Some remarks on the relation to the experimental findings are in order. a) As mentioned earlier, the singular temperature dependence expected from the gauge interaction has not been seen at low temperatures. This is consistent with the fact that the spinon pairing gaps out the $U(1)$ gauge field below a pairing temperature so that various singularities associated with the $U(1)$ gauge field fluctuations do not show up at low temperatures. b) The specific heat does not depend on the applied field up to 8T [16]. This is consistent with the spin-triplet pairing that is not affected by the Zeeman effect. c) When the system becomes superconducting at higher pressure, the Knight shift does not change across the superconducting transition [17]. This can be explained if the resulting superconducting state is related to the spinon pairing state and hence a spin-triplet superconductor.

Previously, Lee et al. [18] proposed a different mechanism for spinon pairing. It is based on the "Amperian pairing" that arises due to the attractive current-current interaction between spinons moving in the same direction. Such an interaction leads to Cooper pairs with a finite center of mass momentum, resulting in a translational-symmetry broken state. The main difference between our proposal and that of Ref. [6] is that our spin-triplet state occurs in the normal BCS channel and corresponds to a uniform ground state. 
We start with the following Hamiltonian, which describes a system of fermionic spinons minimally coupled to a $U(1)$ gauge field $[8,10,11,12,13,14]$ :

$$
\mathcal{H}_{a, f}=\sum_{s} \int d^{2} r f_{s}^{\dagger}(\mathbf{r}) \epsilon(-i \boldsymbol{\nabla}-\mathbf{a}) f_{s}(\mathbf{r})
$$

where $\epsilon(-i \boldsymbol{\nabla}-\mathbf{a})$ is obtained by replacing $\mathbf{p}$ by $(-i \boldsymbol{\nabla}-\mathbf{a})$ in the spinon dispersion $\epsilon(\mathbf{p})$. Here $f^{\dagger}$ and $f$ are the spinon creation and annihilation operators. $s=1,2, \ldots N$ is the "spin" index generalized to $N$ components, and a is the fluctuating gauge field. The bare gauge field Hamiltonian can be taken as $\mathcal{H}_{a}=\frac{1}{2} \int d^{2} \mathbf{r}\left\{g_{0}^{2} \mathbf{e}^{2}(\mathbf{r})+\frac{1}{g_{0}^{2}}[\nabla \times \mathbf{a}(\mathbf{r})]^{2}\right\}$, where $\mathbf{a}$ and $\mathbf{e}=-i \frac{\partial}{\partial \mathbf{a}}$ are canonically conjugate quantum operators, and $g_{0}$ is a bare gauge coupling constant. However, the exact form and parameters of the bare gauge field theory are unimportant, as the coupling to spinons generates more relevant terms. This is pictorially described in Fig. 1.1a, where the dynamics of the field is determined by the particle-hole excitations of spinons via the RPA renormalization. The resulting effective propagator of the transverse gauge field takes the following form

$$
\mathcal{D}_{\alpha \beta}(\omega, \mathbf{q})=P_{\alpha \beta}^{(\mathrm{tr})}(\mathbf{q}) \frac{1}{-i \gamma \omega / q+\chi q^{2}},
$$

where $P_{\alpha \beta}^{(\mathrm{tr})}(\mathbf{q})=\delta_{\alpha \beta}-q_{\alpha} q_{\beta} / q^{2}$, and $\gamma$ and $\chi$ are constants, which determine the Landau damping and the Landau diamagnetic susceptibility of the fermions, respectively. In the large- $N$ limit, one can develop a self-consistent Eliashberg-type [19] theory by using the electronic selfenergy (Fig. 1.1b), $\Sigma(\varepsilon)=i \varepsilon\left|\omega_{0} / \varepsilon\right|^{1 / 3}$ (where $\omega_{0} \sim E_{F}$ is a constant). This leads to a non-Fermi liquid behavior. The self-consistency of the theory implies that (i) The lines in the bosonic and fermionic self-energies in Figs. 1.1a and $1.1 \mathrm{~b}$ can be either thin (bare propagator) or thick (renormalized propagator), leading to the same result. Further renormalization does not change the effective dynamics of the fermions and gauge bosons. (ii) Once the above renormalization is taken into account, the vertex corrections are small (see Fig. 1.2). The latter statement is indeed true in the large- $N$ limit, but only if the momentum transfer is not close to $2 p_{F}$. As pointed out by Altshuler et al. [12], the vertex diverges logarithmically if the momentum transfer is exactly equal to twice the Fermi momentum. The leading logarithms can be summed within the standard parquet technique leading to a power-law divergence of the vertex

$$
\Gamma_{2 p_{F}}(\omega, \mathbf{q})=\frac{\Gamma_{0}}{\left[\left|\omega / E_{F}\right|+c\left(q / 2 p_{F}-1\right)^{3 / 2}\right]^{\sigma}},
$$

where $\Gamma_{0}$ is the value of the vertex far from the $2 p_{F}$-anomaly and $c$ and $\sigma$ are some $N$-dependent constants. In Ref. [12], it was shown that within the large- $N$ treatment, $\sigma \propto 1 / N$. The extrapolation of the results in the large- $N$ limit to $N=2$ leads to $\sigma \approx 0.36$ for the circular Fermi surface. It can be shown that $\sigma$ increases as the curvature of the Fermi surface becomes larger [20]. On the other hand, the small- $N$ approach in Ref. [12] gives $\sigma \approx 0.52$. Clearly, these results provide only an estimate of the exponent $\sigma$ as there is no truly controlled method of treating strong gauge fluctuations.

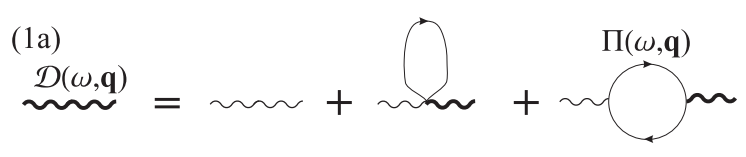

(1b)
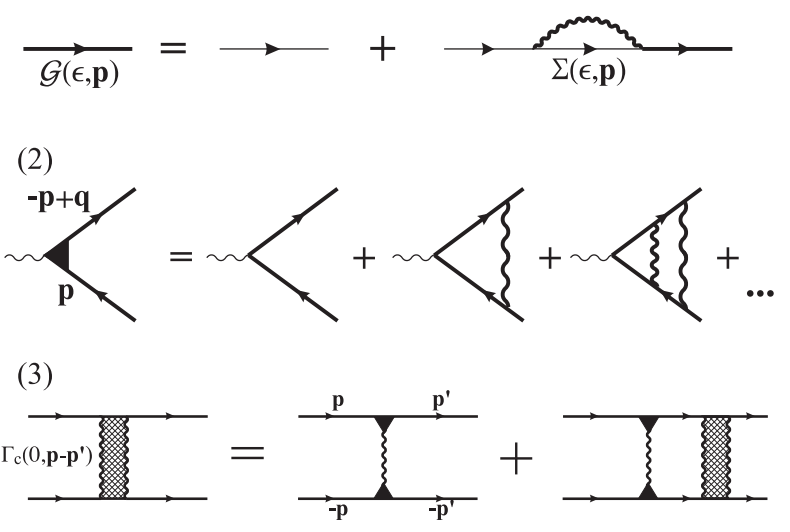

FIG. 1: (1a): The RPA approximation for the gauge-field propagator. (1b): The leading contribution to the fermionic self-energy. (2): The renormalization of the spinon vertex by gauge interactions. The corresponding diagrams diverge logarithmically if the momentum transfer is equal to two Fermi momenta, $q=2 p_{F}$. (3): The Bethe-Salpeter equation for the irreducible part of the scattering amplitude in the Cooper channel. The wavy line includes all interactions, including the magnetic interactions mediated by the gauge field. The shaded vertices represent processes described by Fig. 1.2.

Here we assume that the structure of the theory found at large $N$ is preserved in the physical limit of $N=2$. This implies that the structures of all power laws in the bosonic and fermionic propagators are the same but the parameters of the effective theory can be some non-universal numbers. One of these parameters is the exponent $\sigma$ in Eq. (3). This parameter also enters the temperature dependence of the susceptibility and therefore is a physically observable quantity. Thus instead of describing the effective theory in terms of the unphysical number of flavors $N$, it is perhaps more reasonable to use the parameter $\sigma$ as the variable which "controls" the effective theory. This is the point of view we take in this paper.

We note that the power law divergence (3) can be viewed as a strong renormalization of the Kohn anomaly in the nonFermi liquid phase. A natural question is whether there are instabilities related to this anomaly. Ref. [21] studied possible instabilities in the particle-hole channel and concluded that a strong enough short-range interaction may lead to a density-wave transition in the system. Below we address the issue of possible instabilities in the Cooper channel. The theoretical motivation comes from the following observation: In a usual Fermi liquid, the effective interaction with momen- 
tum $2 p_{F}$, is not divergent, but non-analytic. This weak nonanalyticity is not benign and leads to a number of observable effects such as "non-Fermi-liquid" temperature corrections to thermodynamics and transport coefficients [22, 23, 24]. This anomaly can also give rise to superconducting or pairing instabilities even in the presence of repulsive interactions. The latter phenomenon has been known as the Kohn-Luttinger effect [15, 25, 26]. In a usual Fermi liquid, this effect arises because the effective interaction in real space acquires an oscillatory part (Friedel oscillations), which is a reflection of the Kohn anomaly. Thus there appear attractive regions and electrons can pair up due to this attraction. They however, must be far apart from each other which implies a finite orbital angular momentum of a pair. In a gauge-fermion system, the $2 p_{F}$ anomaly is much stronger than in the ordinary Fermi liquid, thus it is natural to consider the possibility of spinon pairing of Kohn-Luttinger-type.

Fig. 1.3 shows pictorially the Bethe-Salpeter equation for the effective interaction in the Cooper channel. Below we will concentrate on the case when the momentum transfer is of order $2 p_{F}$. The wavy line in Fig. 1.3 is assumed to be a combination of the effective magnetic interaction and short-range repulsive interactions which may include the screened "electric" forces mediated by the gauge field. From now on, we will denote the corresponding interaction as $U\left(2 p_{F}\right)$. It is important to include the singular $2 p_{F}$ vertices in the Bethe-Salpeter equation. The resulting equation has the following form:

$$
\begin{array}{r}
\mathcal{T}_{c}\left(\varepsilon, \varepsilon^{\prime} ; \mathbf{p}, \mathbf{p}^{\prime}\right)=U\left(\varepsilon-\varepsilon^{\prime}, \mathbf{p}-\mathbf{p}^{\prime}\right) \Gamma_{2 p_{F}}^{2}\left(\varepsilon-\varepsilon^{\prime}, \mathbf{p}-\mathbf{p}^{\prime}\right) \\
-T \sum_{\eta} \int_{\mathbf{k}} U(\varepsilon-\eta, \mathbf{p}-\mathbf{k}) \Gamma_{2 p_{F}}^{2}(\varepsilon-\eta, \mathbf{p}-\mathbf{k}) \\
\quad \times \mathcal{G}_{\eta}(\mathbf{k}) \mathcal{G}_{-\eta}(-\mathbf{k}) \mathcal{T}_{c}\left(\eta, \varepsilon^{\prime} ; \mathbf{k}, \mathbf{p}^{\prime}\right),
\end{array}
$$

As usual in the Cooper problem, we assume that the integral on the right-hand side is determined by momenta at the Fermi surface and small frequencies $\eta \rightarrow 0$. Therefore, the momentum dependence of all functions in Eq. (4) reduces to the dependence on a single angular variable (i.e., the angle between two of the following vectors $\mathbf{p}, \mathbf{p}^{\prime}$, and $\mathbf{k}$ ). We therefore can simplify the Bethe-Sapleter equation by performing a standard decomposition into harmonics corresponding to different orbital angular momenta, i.e. $f_{l}=\int_{0}^{\pi} f(\phi) \cos (l \phi) d \phi / \pi$, where $f(\phi)$ is an arbitrary function of the angle. In these notations, we can write Eq. (4) as

$$
\begin{aligned}
& \mathcal{T}_{c}\left(\varepsilon, \varepsilon^{\prime} ; l\right)=U\left(2 p_{F}\right)\left[\Gamma_{2 p_{F}}^{2}\right]\left(\varepsilon-\varepsilon^{\prime}, l\right) \\
& -U\left(2 p_{F}\right) T \sum_{\eta}\left[\Gamma_{2 p_{F}}^{2}\right](\varepsilon-\eta, l) C(\eta) \mathcal{T}_{c}\left(\eta, \varepsilon^{\prime} ; l\right),
\end{aligned}
$$

Here $C(\eta)$ is the Cooperon and we assumed that the interaction (without the vertex corrections) has no singularity at $q=2 p_{F}$. Pairing instability arises in principle only if there is an effective attraction in one of the channels labelled by the orbital angular momentum $l$. In the large- $N$ limit, the parameter $\sigma$ may be small and by performing the Fourier transform of the square of the $2 p_{F}$-vertex, we obtain $(\sigma<1 / 6)$

$$
\left[\Gamma_{2 p_{F}}^{2}\right](0, l)=\frac{2^{6 \sigma} \Gamma_{0}^{2}}{(1-6 \sigma)} B^{-1}(1-3 \sigma+l, 1-3 \sigma-l) \propto \frac{(-1)^{l+1}}{l^{1-6 \sigma}},
$$

where $B(p, q)=\Gamma(p+q) /[\Gamma(p) \Gamma(q)]$ is the beta-function and the last estimate on the right-hand side corresponds to the limit $l \gg 1$. Therefore, for small $\sigma<1 / 6$, the effective interaction is attractive for large even orbital angular momenta. However, in the physical limit of $N=2$, there is no reason for the parameter $\sigma$ to be small. If $\sigma>1 / 6$, the corresponding Fourier transform of the static double-vertex diverges, but it is cut off by the frequency and we get $(\sigma>1 / 6)$

$$
\left[\Gamma_{2 p_{F}}^{2}\right](\omega, l)=\frac{2 \Gamma_{0}^{2} B(1 / 3,2 \sigma-1 / 3)}{3 \pi c^{1 / 3}}(-1)^{l}\left|\frac{E_{F}}{\omega}\right|^{2 \sigma-\frac{1}{3}},
$$

In this regime, the effective interaction is attractive for all odd orbital angualr momenta, $l=1,3,5, \ldots$.

We now address the possibility of a pairing instability due to the effective attraction. In the usual Fermi liquid an attractive interaction automatically implies a pairing instability, which is a consequence of the Cooper logarithmic divergence. It is not so in the fermion-gauge system because the Cooperon divergence is much weaker here than in the Fermi liquid [we recall that $\mathcal{G}_{\eta}(\mathbf{k})=\left(i \eta\left|\omega_{0} / \eta\right|^{1 / 3}-\xi_{\mathbf{p}}\right)^{-1}$ ]: $C(\eta)=\int \frac{d^{2} \mathbf{k}}{(2 \pi)^{2}} \mathcal{G}_{\eta}(\mathbf{k}) \mathcal{G}_{-\eta}(-\mathbf{k})=v \pi /\left(|\eta|^{2 / 3} \omega_{0}^{1 / 3}\right)$, where $v$ is the density of states.

To find an instability, we need to consider the following BCS self-consistency equation

$$
T U\left(2 p_{F}\right) \sum_{\eta}\left[\Gamma_{2 p_{F}}^{2}\right](\varepsilon-\eta, l) C(\eta) \Phi_{l}(\eta)=\Phi_{l}(\varepsilon),
$$

where $l$ is the orbital angular momentum and $\Phi_{l}$ is related to the corresponding pairing amplitude. The existence of a nontrivial solution of the corresponding eigenvalue problem implies a divergence of the resolvent of the integral (if $T=0$ ) or finite difference (if $T \neq 0$ ) equation (5); this can be interpreted as a pairing instability (see also Ref. [26]). To unequivocally establish the existence of a pairing instability for given values of the bare interactions, we look for an infrared divergence in Eq. (8). For this, we assume that the eigenvector is a weakly dependent function of the frequency. With this Ansatz, we get

$$
\left[1+(-1)^{l} v \kappa U\left(2 p_{F}\right) E_{F}^{2 \sigma-2 / 3} \int_{T}^{E_{F}} \frac{d \varepsilon}{|\varepsilon|^{2 \sigma+1 / 3}}\right] \Phi_{l}=0,
$$

where $\kappa=(2 / 3) \Gamma_{0}^{2} B(1 / 3,2 \sigma-1 / 3)\left(E_{F} / \omega_{0}\right)^{1 / 3}$ is just a dimensionless constant, $v=m / \pi$, and we assumed that the Fermi energy serves as a high-energy cut-off. Clearly a non-trivial solution always exists if $l$ is an odd number and $\sigma \geq 1 / 3$. The critical value $\sigma=1 / 3$ gives a logarithmic divergence and the transition temperature $T_{*} \sim E_{F} \exp \left\{-1 /\left[v \kappa U\left(2 p_{F}\right)\right]\right\}$, while for larger values we get a power-law singularity and $T_{*} \sim E_{F}\left[v \kappa U\left(2 p_{F}\right)\right]^{1 /(2 \sigma-2 / 3)}$. 
We note that the transition temperature depends on the orbital angular momentum very weakly (apart from the oddeven dependence); an explicit dependence on the value of $l$ appears only in the subleading orders and enters via the overall cut-off constant. Due to this weak dependence of the transition temperature on $l$, the actual pairing symmetry is nonuniversal and depends on the structure of the effective interaction far from $2 p_{F}$ and/or details of the fermion dispersion. What our argument above explicitly shows is that in the theory with $\sigma \geq 1 / 3$, the system is unstable to the pairing at some odd orbital angular momentum (or momenta). Thus the paired state is a $Z_{2}$ spin liquid [27].

If the pairing occurs in a single odd angular momentum channel, then at some finite temperature of order $T_{*}$ we expect a crossover from the spinon-Fermi surface phase to a pseudogap-like phase as the temperature decreases. On the other hand, the susceptibility and specific heat coefficients seem finite at low temperatures below the "kinks" at a few Kelvin. There may be two possible ways to explain this: a) The low temperature phase is not a homogeneous phase. Various extrinsic effects (such as tiny amount of disorder, structural inhomogeneity, and warping of the spinon Fermi surface) can lead to the suppression of the local transition temperature and the local pairing amplitude in certain regions of real space. The resulting state would consist of the gapless spinon puddles (where the pairing amplitude vanishes) in the sea of a $Z_{2}$ spin liquid. This may be consistent with the observation that two different NMR relaxation times have been observed [3, 4]. The finite susceptibility and specific heat coefficient at low temperatures (below the "kink" temperature) may be due to the gapless regions. b) Another possibility is that the pairing occurs in a large orbital angular momentum channel or the pairing amplitude is given by a superposition of contributions from multiple angular momentum channels. Then a large number of nodal excitations may exist and practically it may look like there is an extended gapless region in momentum space. Which scenario is realized in real materials may depend on non-universal physics and it requires more detailed analysis of the band dispersion and sub-dominant interactions in the Cooper channel. This is beyond the scope of this paper, but it may be an excellent topic of future study.

In conclusion, we demonstrated that there exist KohnLuttinger-like pairing instabilities in the $U(1)$ spin liquid with a spinon Fermi surface. This pairing occurs in odd-angularmomentum/spin-triplet channels. As explained in the introduction, this is consistent with several experimental findings. On the other hand, the previous proposal of the "Amperian pairing" [6] (while it explains various aspects of the same experiments) suggests a spin-singlet pairing and a spatially modulated pairing amplitude with a well defined wavevector. This corresponds to an incommensurate version of the valence bond solid phase and would induce lattice distortion. Thus the difference between two proposals may be tested by X-ray scattering.

Finally we emphasize that the microscopic scenario presented here may be relevant not only to the gauge-fermion system of spinons but also to other fermionic systems with singular interactions, which include electrons near an Ising ferromagnetic instability or near a nematic ordering quantum critical point [28] and the vortex metal phase proposed in Refs. [29, 30, 31].

The authors are grateful to A. Chubukov, O. Motrunich, and G. Refael for discussions about this work. This research was supported in part by the NSF under Grant No. PHY0551164. It was also supported by the NSERC, CRC, CIFAR, and KRF-2005-070-C00044 (YBK). The authors acknowledge the KITP for hospitality, where most of this work was performed.

[1] I. Y. Pomeranchuk, Sov. Phys. JETP Lett. 11, 226 (1941).

[2] P. W. Anderson, Science 235, 1196 (1987).

[3] Y. Shimizu, K. Miyagawa, K. Kanoda, M. Maesato, and G. Saito, Phys. Rev. Lett. 91, 107001 (2003).

[4] Y. Kurosaki, Y. Shimizu, K. Miyagawa, K. Kanoda, and G. Saito, Phys. Rev. Lett. 95, 177001 (2005).

[5] O. I. Motrunich, Phys. Rev. B 72, 045105 (2005).

[6] S.-S. Lee and P. A. Lee, Phys. Rev. Letts. 95, 036403 (2005).

[7] A. M. Polyakov, Phys. Lett. 59, 82 (1975).

[8] L. B. Ioffe and A. I. Larkin, Phys. Rev. B 39, 8988 (1989).

[9] M. Hermele, T. Senthil, M. P. A. Fisher, P. A. Lee, N. Nagaosa, and X.-G. Wen, Phys. Rev. B 70, 214437 (2004).

[10] C. Nayak and F. Wilczek, Nucl. Phys. B 417, 359 (1994).

[11] Y. B. Kim, A. Furusaki, X. G. Wen, and P. A. Lee, Phys. Rev. B 50, 17917 (1994).

[12] B. L. Altshuler, L. B. Ioffe, and A. J. Millis, Phys. Rev. B 50, 14048 (1994).

[13] J. Polchinski, Nucl. Phys. B 422, 617 (1994).

[14] V. M. Galitski, Phys. Rev. B 72, 214201 (2005).

[15] W. Kohn and J. M. Luttinger, Phys. Rev. Lett. 15, 524 (1965).

[16] Y. Nakazawa et al. (unpublished).

[17] Y. Shimizu et al. (to be published).

[18] S.-S. Lee, P. A. Lee, and T. Senthil, Phys. Rev. Lett. 98, 067006 (2007).

[19] G. M. Eliashberg, Sov. Phys. JETP 11, 696 (1960) [Zh. Eksp. Teor. Fiz. 38, 966 (1960)].

[20] Y. B. Kim and A. J. Millis, Phys. Rev. B 67, 085102 (2003).

[21] B. L. Altshuler, L. B. Ioffe, A. I. Larkin, and A. J. Millis, Phys. Rev. B 52, 4607 (1995).

[22] V. M. Galitski and S. Das Sarma, Phys. Rev. B 70, 035111 (2004).

[23] V. M. Galitski, A. V. Chubukov, and S. Das Sarma, Phys. Rev. B 71, 201302 (2005).

[24] A. V. Chubukov, D. L. Maslov, S. Gangadharaiah, , and L. I. Glazman, Phys. Rev. B 71, 205112 (2005).

[25] A. V. Chubukov, Phys. Rev. B 48,1097-1104 (1993).

[26] V. M. Galitski and S. Das Sarma, Phys. Rev. B 67, 144520 (2003).

[27] X.-G. Wen, Phys. Rev. B 65, 165113 (2002).

[28] J. Rech, C. Pepin, and A. Chubukov, Phys. Rev. B 74, 195126 (2006).

[29] V. M. Galitski, G. Refael, M. P. A. Fisher, and T. Senthil, Phys. Rev. Lett. 95, 077002 (2005).

[30] J. Alicea, O. Motrunich, M. Hermele, and M. P. A. Fisher, Phys. Rev. B 72, 064407 (2005).

[31] N. Lindner, A. Auerbach, and D. Arovas, arXiv:condmat/0701571 (2007). 